\title{
A truncation allele in vascular endothelial growth factor c reveals distinct modes of signaling during lymphatic and vascular development
}

\author{
Jacques A. Villefranc ${ }^{1}$, Stefania Nicoli ${ }^{1, *}$, Katie Bentley ${ }^{2, \neq}$, Michael Jeltsch ${ }^{3}$, Georgia Zarkada ${ }^{3}$, John C. Moore ${ }^{1}$, \\ Holger Gerhardt ${ }^{2}$, Kari Alitalo ${ }^{3}$ and Nathan D. Lawson ${ }^{1, \S}$
}

\begin{abstract}
SUMMARY
Vascular endothelial growth factor C (Vegfc) is a secreted protein that guides lymphatic development in vertebrate embryos. However, its role during developmental angiogenesis is not well characterized. Here, we identify a mutation in zebrafish vegfc that severely affects lymphatic development and leads to angiogenesis defects on sensitized genetic backgrounds. The um18 mutation prematurely truncated Vegfc, blocking its secretion and paracrine activity but not its ability to activate its receptor Flt4. When expressed in endothelial cells, vegfc ${ }^{u m 18}$ could not rescue lymphatic defects in mutant embryos, but induced ectopic blood vessel branching. Furthermore, vegfc-deficient endothelial cells did not efficiently contribute to tip cell positions in developing sprouts. Computational modeling together with assessment of endothelial cell dynamics by time-lapse analysis suggested that an autocrine Vegfc/Flt4 loop plays an important role in migratory persistence and filopodia stability during sprouting. Our results suggest that Vegfc acts in two distinct modes during development: as a paracrine factor secreted from arteries to guide closely associated lymphatic vasculature and as an autocrine factor to drive migratory persistence during angiogenesis.
\end{abstract}

KEY WORDS: Vegfc, Angiogenesis, Lymphatic, Zebrafish

\section{INTRODUCTION}

Evolutionarily conserved transport systems comprise blood and lymphatic vessels that are lined by distinct endothelial cell types. Blood vessels allow circulation of blood cells, gas exchange and delivery of metabolic products to and from tissues. The lymphatic system consists of blind-ended vessels that return fluid and macromolecules from interstitial tissue space back into blood circulation, while serving as a conduit for the development and transport of lymphoid cells (Tammela and Alitalo, 2010). During embryogenesis, blood vessels arise from endothelial cell progenitors that coalesce and differentiate into arteries and veins (Cleaver and Krieg, 2010). Subsequently, new blood vessels sprout from preexisting ones by angiogenesis (Poole and Coffin, 1989). Later, lymphatic progenitors are specified from endothelial cells in the embryonic cardinal vein (Srinivasan et al., 2007), which sprout to form lymphatic vessels.

Vascular endothelial growth factor C (Vegfc) is the central regulator of lymphatic development (Tammela and Alitalo, 2010). Vegfc is expressed as a pro-peptide and cleaved to yield a secreted factor that acts on lymphatic endothelial cells (Joukov et al., 1997). Ectopic vegfc expression can cause lymphatic hyperplasia (Jeltsch et al., 1997) and mice or zebrafish lacking vegfc fail to develop

\footnotetext{
${ }^{1}$ Program in Gene Function and Expression, University of Massachusetts Medical School, Worcester, MA 01605, USA. ${ }^{2}$ Cancer Research UK, London Research Institute, Lincoln's Inn Fields, London WC2A 3LY, UK. ${ }^{3}$ Molecular Cancer Biology Program, Biomedicum Helsinki, Haartman Institute, University of Helsinki, Haartmaninkatu 8 (PO Box 63), FI-00014, Helsinki, Finland.

*Present address: Department of Medicine, Section of Cardiology, Yale University School of Medicine, New Haven, CT 06511, USA

${ }^{\ddagger}$ Present address: Computational Biology Laboratory, Centre for Vascular Biology Research, Beth Israel Deaconess Medical Center, Harvard Medical School, 99 Brookline Avenue, RN 231, Boston, MA 02215, USA

${ }^{\S}$ Author for correspondence (nathan.lawson@umassmed.edu)
}

Accepted 23 January 2013 lymphatic vessels and exhibit a block in primary sprouting of lymphatic progenitors from the cardinal vein (Karkkainen et al., 2004; Küchler et al., 2006; Yaniv et al., 2006). Vegfc functions primarily through binding and activation of the Vegfr3 receptor tyrosine kinase (Joukov et al., 1996) (hereafter referred to as Flt4), which is specifically expressed in both blood vascular and lymphatic endothelial cells (Kaipainen et al., 1995; Kukk et al., 1996). While Vegfc can also activate Vegfr2, a Flt4-specific form of Vegfc can induce lymphatic hyperplasia (Veikkola et al., 2001). Vegfc and its receptor are also important for lymphatic homeostasis in adults. Notably, mutations in human FLT4 that reduce kinase activity cause congenital lymphedema (Karkkainen et al., 2000). Thus, Vegfc/Flt4 signaling is essential for lymphatic development and homeostasis in vertebrates.

In addition to their roles in lymphatic development, Vegfc and Flt4 have been implicated in angiogenesis. In the circulatory system, flt 4 is expressed in actively sprouting endothelial cells, where it becomes restricted to tip cells (Siekmann and Lawson, 2007; Tammela et al., 2008) and loss of flt4 in mouse or zebrafish causes defects in angiogenesis (Covassin et al., 2006; Dumont et al., 1998; Tammela et al., 2011; Tammela et al., 2008). Interestingly, flt 4 is a central target of repression by the Notch pathway during angiogenesis. Notch activation downregulates flt4 expression (Lawson et al., 2001), whereas Notch deficiency causes increased and ectopic flt4 expression (Siekmann and Lawson, 2007; Tammela et al., 2008). Furthermore, reduced flt4 signaling attenuates the hyperangiogenic phenotype associated with loss of Notch (Benedito et al., 2012; Siekmann and Lawson, 2007). However, the role of vegfc during angiogenesis is less clear. Although transgenic overexpression of Vegfc in mice can induce lymphatic hyperplasia (Jeltsch et al., 1997), it also induced angiogenesis, which was restricted to embryonic blood vessels that expressed Flt4 and was caused by overexpressing Vegfc in endothelial cells themselves (Lohela et al., 2008). Furthermore, 
$v e g f c$ is normally expressed in endothelial cells during development (Covassin et al., 2006; Tammela et al., 2008), suggesting that a Vegfc/Flt4 autocrine loop might be important for angiogenesis.

Here, we describe a zebrafish mutant, um 18, that fails to develop lymphatic vessels and displays mild angiogenesis defects on a sensitized genetic background. The um 18 mutation caused premature truncation of zebrafish Vegfc, which hindered its secretion but not its ability to activate Flt4. Vegfc ${ }^{\text {um } 18}$ could not rescue lymphatic formation, but caused ectopic blood vessel branching when expressed in endothelial cells. Additionally, vegfcdeficient cells displayed autonomous defects in tip cell potential, suggesting an important autocrine role in angiogenesis. Computational modeling and time-lapse analysis of angiogenesis in Vegfc-deficient embryos suggested an important role in maintaining filopodia stability and migratory persistence. Together, our results suggest that Vegfc signals via distinct autocrine and paracrine mechanisms to pattern blood and lymphatic vessels, respectively.

\section{MATERIALS AND METHODS}

\section{Zebrafish handling and maintenance}

Zebrafish were maintained in accordance with approved University of Massachusetts Medical School IACUC protocols. Tg(flila:egfp $)^{y 1}, k d r l^{y 17}$ and $T g(k d r l: \text { rasmcherry })^{s 89}$ lines have been described elsewhere (Covassin et al., 2006; Herbert et al., 2009; Lawson and Weinstein, 2002).

\section{Antibodies}

A glutathione-S-transferase (GST)-fusion protein containing the first 225 amino acids of zebrafish Fli1b was expressed in BL21 E. coli, purified on glutathione-Sepharose beads (GE Healthcare) and cleaved from the GST using PreScission Protease (GE Healthcare). Flt4 peptide (NH2CASDELERFEHKHRGA; New England Peptide, Gardner, MA, USA) or Fli1b protein was used to raise rabbit polyclonal antisera (Capralogics, Hardwick, MA, USA)

\section{Identification and cloning of um18}

N-ethyl-N-nitrosourea (ENU) mutagenesis and haploid screening were performed as described previously (Covassin et al., 2009), except that embryos were screened for loss of primordial hindbrain channel (PHBC). F1 females were outcrossed to TL males and progeny from individual F2 incrosses were assayed for PHBC formation at 26 hours post-fertilization (hpf) and for thoracic duct (TD) at 7 days post-fertilization (dpf). Embryos that failed to form both were designated mutants and used for linkage analysis as described previously (Lawson et al., 2003). To assess linkage to vegfc, we used CA markers (Z25069, Z11618, Z26040, Z24128) and a single-nucleotide polymorphism (SNP) in the vegfc $3^{\prime}$ UTR (see supplementary material Table $\mathrm{S} 1$ for primer sequences) that creates an $\mathrm{XcmI}$ site. The um 18 mutation was identified by sequencing PCR products encompassing vegf $c$ exons amplified from genomic DNA. To genotype the vegf $c^{u m 18}$ allele, we used a customized TaqMan SNP assay (Applied Biosystems). The $k d r^{y 17}$ allele was genotyped as previously (Covassin et al., 2006).

\section{Phenotypic analysis}

General morphology and circulation were assessed using transmitted light on an MZ FLIII stereomicroscope (Leica). Images were recorded on an AxioCam MRc digital camera (Zeiss). Vascular morphology in $T g(f l i l a: e g f p)^{y l}$ embryos was imaged using a Leica DMIRE2 confocal microscope (HC PL APO 20×/0.70 CS objective). Microangiography was performed as described previously (Isogai et al., 2001). Intersomitic vessel (ISV) connections were scored as arterial or venous based on flow direction and counted from the fifth to the last somite at $72 \mathrm{hpf}$. ISV length was determined in live or fixed embryos subjected to GFP immunostaining as described (Covassin et al., 2006). To quantify endothelial nuclei, embryos were fixed in $2 \%$ paraformaldehyde overnight at $4{ }^{\circ} \mathrm{C}$ and permeabilized with $0.5 \%$ Triton X-100 in PBS for 30 minutes. Samples were incubated in blocking solution (PBS containing $0.1 \%$ Triton X-100, 10\% normal goat serum, $1 \%$ BSA, $0.01 \%$ sodium azide), followed by Fli1b antiserum at 1:1000 in blocking solution. Embryos were washed four times for 15 minutes each, followed by an overnight wash at $4{ }^{\circ} \mathrm{C}$ and staining with goat anti-rabbit Alexa-Fluor 568 antibody (Molecular Probes) at 1:1000 in blocking solution. We counted nuclei in three adjacent ISVs and dorsal longitudinal anastomotic vessels in embryos injected with $15 \mathrm{ng}$ control MO or Dll4 MO (Siekmann and Lawson, 2007). Ectopic branching points were counted for the same ISVs.

\section{Analysis of Vegfc knockout mouse embryos}

Mouse studies were approved by the Committee for Animal Experiments of the District of Southern Finland. $V e g f c+/ l a c Z$ mice are described elsewhere (Karkkainen et al., 2004). Embryos were staged according to Edinburgh Mouse Atlas criteria, harvested at E9.5, fixed in 4\% paraformaldehyde, washed in PBS and processed for whole-mount staining as described previously (Tammela et al., 2011). Endomucin staining (sc-65495, Santa Cruz), followed by Alexa Fluor 488-conjugated anti-rat antibody (Molecular Probes), was used to visualize blood vessels in the embryos. Samples were mounted with Vectashield mounting medium (Vector Labs) containing 4,6-diamidino-2-phenylindole (DAPI) (H-1200, Vector Laboratories) and analyzed with a confocal microscope (Zeiss LSM 510 Meta, $10 \times$ objective with NA 0.45 ). Three-dimensional projections were digitally reconstructed from confocal $z$-stacks. Branching points in cranial vessels and ISV length between somites 14 and 16 were manually counted using ImageJ software (NIH; version 1.43r). Statistical analysis was carried out with PASW Statistics 18.0 software (SPSS). For comparison of means a two-way ANOVA test followed by Tukey's HSD post-hoc test were used. Data are presented as mean \pm s.e.m. with statistical significance indicated.

\section{Cloning and plasmid construction}

Zebrafish vegfc, vegf $c^{u m 18}$ and $v e g f c^{s s}$ middle entry clones (pMEveg $f c$, pMEvegf $c^{\text {um } 18}$, pMEvegf $c^{s s}$ ) lacking stop codons were constructed by Gateway cloning as described (Villefranc et al., 2007) (see supplementary material Table S1 for primer sequences). pMEvegfc, pMEveg $c^{u m l 8}$, pMEvegf ${ }^{s s}$ or pMEegfp3 (Villefranc et al., 2007) were used in LR reactions with pDestTol2pA, p5Efliebs and p3E-2Amcherry to yield pTol2fliebs:vegfc-2Amcherry, pTol2fliebs:vegfc ${ }^{\text {um18 }}$-2Amcherry, pTol2fliebs:vegfc ${ }^{s s}$-2Amcherry and pTol2fliebs:egfp-2Amcherry. To generate mammalian expression constructs, pMEvegfc or pMEvegfc ${ }^{\text {um } 18}$ were used in an LR reaction with pCSDest2 (Villefranc et al., 2007) and p3E-2Amcherry to produce pCSvegfc-2Amcherry and pCSvegfc $c^{\text {um } 18}$ 2Amcherry. pMT-Ex-zfVegfc-wt and pMT-Ex-zfVegfc ${ }^{\text {um } 18}$ were constructed by cloning a $B a m \mathrm{HI} / N o t \mathrm{I}$-cleaved PCR product of zebrafish vegfc into pMTBiP-V5His-C (Invitrogen), which was modified to include a hygromycin cassette and a frameshift mutation between the BiP signal peptide and the multiple cloning site such that the $5^{\prime}$-GAT-3' from the BglII site codes for an aspartic acid residue. A human VEGFC ${ }^{\text {um } 18}$ middle entry vector was constructed as above using the primers listed in supplementary material Table S1. pME-VEGFC ${ }^{\mathrm{um} 18}$ was LR cloned into pCSDest (Villefranc et al., 2007) to create pCS-VEGFC ${ }^{u m 18}$. To generate pCSflt4$h a$, we created pMEflt 4 lacking its endogenous stop codon (supplementary material Table S1). pMEflt4 and p3E-HA were LR cloned into pCSDest 2 to give pCS-flt4-ha (Villefranc et al., 2007).

\section{Transient transgenic vegfc expression}

We co-injected 25 pg pTol 2 constructs into one-cell stage embryos with 25 pg tol 2 transposase mRNA. To assay ISV defects, we injected wild-type $\operatorname{Tg}(f l i l a: e g f p)^{y l}$ embryos and counted ectopic Cherry-positive vessel branches in six hemisegments at $30 \mathrm{hpf}$. For rescue experiments, we injected embryos from an incross of $\mathrm{Tg}(\text { flila:egfp })^{y l}$; veg $\mathrm{fc}^{+/ u m l 8}$ carriers. Injected embryos with Cherry-positive vessels were scored for TD formation at 5 dpf and genotyped for um 18 .

\section{Production of Vegfc-conditioned media}

S2 cells were transfected with either pMT-Ex-zfVegfc or pMT-Ex$\mathrm{zfVegfc}^{\mathrm{um} 18}$ and stable cell pools were created according to standard protocols. To produce protein, cells were induced with $1 \mathrm{mM} \mathrm{CuSO}_{4}$ and conditioned cell supernatant was harvested 3.5 days post-induction. 


\section{Yolk membrane angiogenesis assay}

Four nanoliters of Vegfc-, Vegfc ${ }^{\mathrm{um} 18}$ - or negative control-conditioned medium was injected into the perivitelline space of anesthetized 48-hpf $\operatorname{Tg}(f l i l a: e g f p)^{y 1}$ embryos as previously (Nicoli et al., 2009). Ectopic subintestinal vessels (SIVs) were visualized by confocal microscopy and counted at $3.5 \mathrm{dpf}$. For xenografts, NIH3T3 cells were transfected with $1 \mu \mathrm{g}$ pCSvegfc-2Amcherry or pCSvegfciml8-2Amcherry using Lipofectamine 2000 (Invitrogen), grown for 48 hours, and subjected to fluorescenceactivated cell sorting (FACS) to isolate expressing cells. Approximately 6000 Cherry-positive cells were diluted in Matrigel (Sigma) and injected into the perivitelline space as above. Xenografts were imaged at 2 days postengraftment by confocal microscopy. Imaris (Bitplane) was used to quantify the relative number of $v e g f c$-expressing cells (red voxels) and vessels (green voxels) as described previously (Nicoli et al., 2010).

\section{Analysis of VEGFC secretion}

NIH3T3 cells were transfected with $1 \mu \mathrm{g}$ pSport6CMV-VEGFC (Open Biosystems) or pCS-VEGFC ${ }^{\text {um } 18}$ using Lipofectamine 2000. Medium was collected at 30 and 72 hours post-transfection and concentrated using centrifugal filter units (Ultragel 10K, Millipore) while cells were lysed in RIPA buffer (150 mM NaCl, $50 \mathrm{mM}$ Tris $\mathrm{pH} 8.0,1 \% \mathrm{NP} 40,0.1 \%$ SDS) containing protease inhibitors (Complete Mini, Roche). Blots were probed with a polyclonal human anti-VEGFC antibody (Joukov et al., 1996), stripped and reprobed with a monoclonal anti-Tubulin antibody (kindly provided by Dr Michael Green, University of Massachusetts Medical School, Worcester, MA, USA).

\section{Flt4 activation assay}

NIH3T3 cells were transiently transfected with $1 \mu \mathrm{g}$ pCSflt4-HA. After 24 hours, cells were starved for 24 hours and stimulated for 7 minutes with zebrafish Vegfc-, Vegfc ${ }^{\text {um } 18}$ - or negative control-conditioned medium. Cells were lysed in RIPA buffer containing phosphatase and protease inhibitors and used for immunoprecipitation using zebrafish Flt4 polyclonal antibody. Immunoprecipitates were subjected to SDS-PAGE and western blotting. Blots were probed with a monoclonal antibody against phosphotyrosine (4G10 clone, Millipore), stripped, and reprobed with monoclonal anti-HA antibody (BABCO).

\section{Mosaic analysis}

Transplantations were performed as described (Covassin et al., 2009). Donors were injected with control (5 ng) or Vegfc MO (2.5 and $5 \mathrm{ng})$. Alternatively, cells were taken from embryos derived from an incross of $T g(f l i 1: e g f p)^{y l}$; vegf $c^{u m 18}$ carriers and donors were genotyped following transplantation. At $30 \mathrm{hpf}$, the location of donor cells in host vessels was calculated and analyzed using a Fisher's exact test as described previously (Nicoli et al., 2012).

\section{Computational modeling}

The memAgent-Spring model (MSM) (Bentley et al., 2008; Bentley et al., 2009) was used to investigate four possible autocrine effects of Vegfc/Flt4 signaling to identify the best match to experimental data. The MSM has previously been calibrated to match wild-type sprouting data; therefore, Vegfc/Flt4 signaling is encompassed in existing parameters. We modeled the loss of Vegfc by a reduction in an existing model parameter. For each mechanism simulated, a single parameter $k$ was created to control the strength of this loss, under four different conditions as follows:

Type 1 - Vegfc directly affects D114 and migration. (a) Vegfc/Flt4 acts to improve signaling via Vegfr 2 by a constant amount. For MO, $k$ is a small fixed amount subtracted from the total local VEGF of a memAgent. (b)
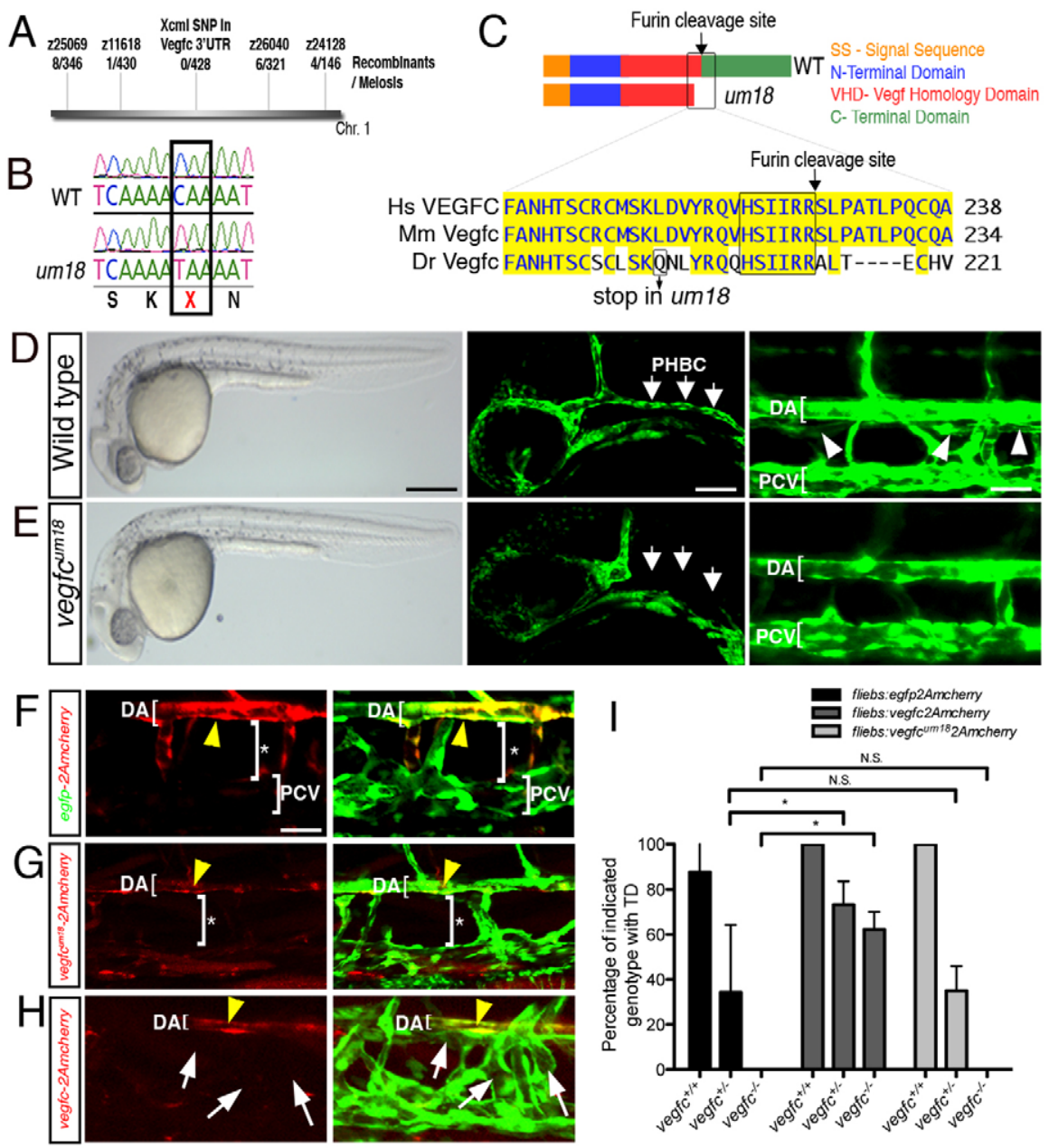

Fig. 1. vegfc ${ }^{u m 18}$ mutants display defects in vein and lymphatic vessel development. (A) Linkage map of markers on zebrafish chromosome 1 used in this study. (B) um 18 mutation in vegfc exon 4 . Box indicates the Gln202 to stop transition. (C) Vegfc domains and amino acid alignment near the um 18 truncation. Hs, human; Mm, mouse; Dr, zebrafish. (D,E) (Left) Transmitted light images of embryos at $30 \mathrm{hpf}$. (Middle) Confocal micrographs of head blood vessels at $30 \mathrm{hpf}$. Primordial hindbrain channel (PHBC) is indicated by arrows. (Right) Confocal micrographs of trunk vessels at $5 \mathrm{dpf}$. Thoracic duct (TD) is indicated by arrowheads. Wild-type (D) and vegfc ${ }^{\text {um } 18}$ mutant (E) Tg(fli1a:egfp $)^{y 1}$ siblings are shown. (F-H) Confocal micrographs of Tg(fli 1a:egfp) $)^{y}$ vegfc ${ }^{\text {um } 18}$ mutant embryos COinjected with $25 \mathrm{pg}$ to/2 transposase mRNA and 25 pg (F) pTol2-fli1ebs:egfp-2Amcherry, (G) pTol2flitebs:vegfc ${ }^{\text {um18}}$-2Amcherry or $(\mathrm{H})$ pTol2flipebs:vegfc-2Amcherry. Yellow arrowheads indicate arterial endothelial cells expressing mCherry. Bracket with asterisk indicates absence of TD. White arrows indicate TD rescue. (D-H) Lateral view, anterior to the left, dorsal up. (I) Percentage of embryos showing TD of the indicated genotype injected with the indicated constructs. Values are the average of three independent experiments. ${ }^{*} P<0.05$; N.S., not significant; error bars indicate s.e.m. DA, dorsal aorta; PCV, posterior cardinal vein. Scale bars: $250 \mu \mathrm{m}$ in D,E left; $50 \mu \mathrm{m}$ in D,E middle; $25 \mu \mathrm{m}$ in $\mathrm{D}$, E right, $\mathrm{F}-\mathrm{H}$. 
Vegfc/Flt4 signaling proportionally reduces Vegfr2 signaling. For MO, $k$ is the proportion of the total local VEGF of a memAgent removed.

Type 2 - Vegfc indirectly affects D114 but directly affects migration. (a) Vegfc/Flt4 induces initiation of filopodia. For MO, $k$ is the probability of a filopodia extending in response to VEGF stimulation. (b) Vegfc/Flt4 promotes persistence and stability of filopodia. For MO, $k$ is the probability of a filopodium to randomly begin retracting.

In each case, $k$ was directly calibrated using a sensitivity analysis to match transplant data using the approach below (see supplementary material Table S2 for parameter values). If a matching parameter could be found, each mechanism was then tested in a simulation modeling the dorsal migration of a tip cell through the somites, as defined below. Type $1 \mathrm{~b}$ and Type $2 \mathrm{~b}$ matched the chimeric data, but only Type $2 \mathrm{~b}$ was able to match the ISV extension data.

\section{Modeling chimeric transplant data}

To model transplant data, embryos were considered as a single simulation of a vessel comprising ten cells in a continuous gradient of Vegfa, linearly increasing in the $y$-axis. A simple uniform, random contribution of Vegfcdeficient cells to the vessel was capped at a maximum of $50 \% \mathrm{MO}$ cells to control cells. Hence, some 'embryos' could have zero MO cells and a maximum of five in random positions. Only embryos that contained at least one MO cell were counted. The same method was used to quantify chimeric simulations as was used experimentally.

\section{Modeling ISV tip cell extension}

To simulate ISV migration, a version of the model was implemented to focus on measuring the distance reached by a traveling tip cell. In this case, Dl14/Notch signaling was switched off as the selection process was assumed to have occurred already and a tip cell selected. A linear, increasing gradient of Vegfa was created in a column of three-dimensional space, $10 \mu \mathrm{m}$ wide in the center above the tip cell, reaching up for $100 \mu \mathrm{m}$. Concentration settings were as previously described (Bentley et al., 2009): 0.04 molecules of VEGF multiplied by the $y$-axis position in each grid site. This assumes that VEGF is not accessible in somites to the left or right of the column and thus the tip will not grow into somite space.

\section{Time-lapse analysis}

$T g(f l i l a: e g f p)^{y 1}$ embryos were injected with $5 \mathrm{ng}$ control or Vegfc MO and mounted in agarose at $16 \mathrm{hpf}$. Images of control and morphant embryos were captured in parallel on a LSM7 MP laser-scanning microscope (Zeiss) equipped with a Chameleon Ti:Sapphire pulsed laser (Coherent) every 5 minutes over 8 hours using ZEN 2009 software with the Multi-time macro (Zeiss). Time-lapse series were loaded into ImageJ to analyze ISV sprouting and filopodial extension/retraction. ISV length (from the base of the dorsal aorta to the leading edge of the ISV) and filopodia length were measured manually in each frame using ImageJ. We measured the lengths of at least ten filopodia from five separate embryos at 5-minute intervals for a 60 minute span. Filopodia measurements were made on ISVs of comparable length in control- and Vegfc MO-injected embryos. For ISV length, we limited analysis to ISVs that were between 3 and $6 \mu \mathrm{m}$ within the first few movie frames. We normalized the starting time of each ISV sprout to their first detectable length and recorded length every 5 minutes for a total of 300 minutes. For ISV forward and retraction rates, we calculated the change in distance between successive time points from 0 to $30 \mu \mathrm{m}$ in length for 16 ISVs from eight embryos (control MO) or 17 ISVs from six embryos (Vegfc MO). Negative (retraction) and positive (forward) values for each ISV were analyzed for frequency and averaged. Statistical significance was measured using a paired $t$-test (ISV length) or a Wilcoxon test (retraction/forward rates).

\section{RESULTS}

\section{A truncated form of vegfc perturbs lymphatic development}

A screen for zebrafish embryos lacking a primordial hindbrain channel (PHBC) (supplementary material Fig. S1) identified the um 18 mutant, which is linked to the vegfc locus (Fig. 1A). Analysis of vegfc coding sequence in um 18 mutants revealed a CAA

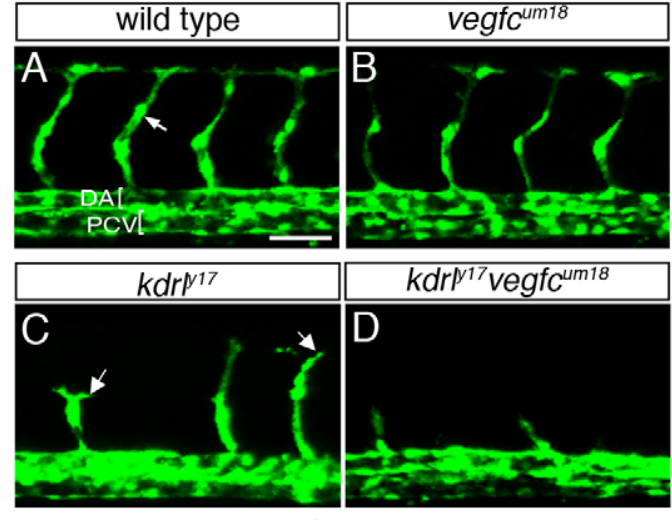

E

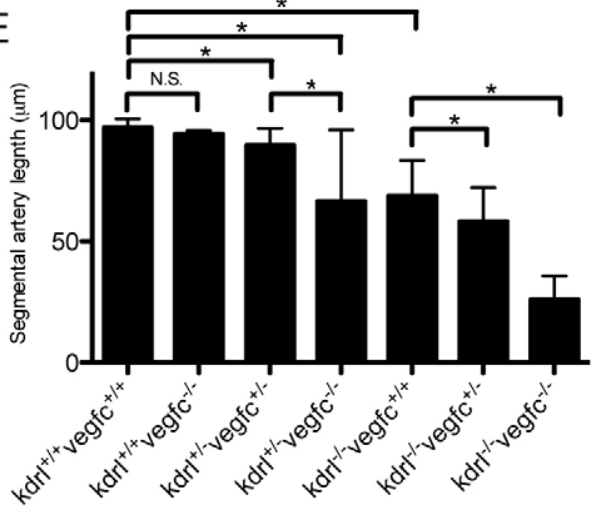

Fig. 2. vegfc ${ }^{u m 18}$ enhances angiogenesis defects in $k d r r^{y 17}$ mutant embryos. (A-D) Confocal micrographs of trunk blood vessels in Tg(fli1a:egfp)y (A) wild-type, (B) vegfc ${ }^{\text {um }^{18}}$ mutant, (C) $k d r^{y^{17}}$ mutant and (D) $k d r^{17}$ vegfc ${ }^{u m 18}$ double-mutant zebrafish embryos at $30 \mathrm{hpf}$. Arrows indicate intersomitic vessels (ISVs). Anterior is to the left, dorsal is up. (E) ISV length in embryos of the indicated genotype at $30 \mathrm{hpf}$. Values are the average from two clutches of embryos. ${ }^{*} P<0.05$; N.S., not significant; error bars indicate s.e.m. DA, dorsal aorta; PCV, posterior cardinal vein. Scale bar: $50 \mu \mathrm{m}$

(Gln202) to TAA (stop) transition (Fig. 1B) that truncates Vegfc 13 amino acid residues upstream of a conserved cleavage site recognized by the proprotein convertase furin (Fig. 1C) (Joukov et al., 1997; Siegfried et al., 2003). Despite the presence of a premature stop codon, we did not observe a decrease in vegfc transcript levels in mutant embryos (data not shown). At $30 \mathrm{hpf}$, wild-type and mutant siblings were generally indistinguishable (Fig. 1D,E, left). However, vegfc $c^{\text {um } 18}$ mutants displayed a partially penetrant defect in PHBC formation (Fig. 1D,E, middle; supplementary material Table S3), which recovered by $40 \mathrm{hpf}$ (data not shown), and fully penetrant defects in lymphatic development. Both heterozygous and homozygous vegfc $c^{\text {um } 18}$ mutant embryos failed to form a thoracic duct (TD), which is the first lymphatic vessel in zebrafish embryos, at 5 dpf (Fig. 1D,E, right; supplementary material Table S3, Fig. S2). We detected vegf $c^{u m 18 /+}$ carriers at expected frequencies in adults, suggesting that they could recover from this defect (data not shown). We also noted loss of parachordal lymphangioblasts, which give rise to the TD (Küchler et al., 2006; Yaniv et al., 2006), at $2 \mathrm{dpf}$ in vegfc $c^{u m} 18$ mutant and heterozygous embryos (supplementary material Fig. S3A-E). In addition, vegfc $c^{u m 18}$ mutants had fewer intersomitic vein connections than wild-type siblings (supplementary material Fig. S3F-H and Table S4), suggesting an early defect in venous sprouting of lymphatic progenitors. 
To confirm that defects in $u m 18$ mutants were due to loss of $v e g f c$, we performed rescue experiments. Since vegfc is normally expressed in the dorsal aorta (Covassin et al., 2006), we drove transgenic endothelial expression using an enhancer from the flila gene (Villefranc et al., 2007). To visualize expressing cells, veg $f c$ coding sequences were fused to mcherry separated by a viral $2 \mathrm{~A}$ peptide sequence in order to express both proteins (Provost et al., 2007). Mutant embryos expressing egfp-2Amcherry or vegfc $c^{u m 18}$ 2 Amcherry in arterial endothelial cells failed to form a TD (Fig. 1F,G,I). By contrast, vegfc-2Amcherry rescued TD formation in vegf $c^{\text {um } 18}$ mutants (Fig. 1H,I). Interestingly, a furin-resistant form of Vegfc $\left(\operatorname{Vegfc}^{\mathrm{ss}}\right)$, which prevents its cleavage and retains the Cterminus, can partially rescue lymphatic formation in vegfc $c^{\text {um } 18}$ mutants (supplementary material Fig. S4A,B). Together, these observations suggest that the C-terminal domain of Vegfc is essential for lymphatic development.

\section{The vegfc ${ }^{u m 18}$ mutation has a weak effect on angiogenesis}

Morpholino (MO)-mediated knockdown of zebrafish Vegfc or Flt4 causes a mild, partially penetrant block in intersomitic vessel (ISV) formation (Covassin et al., 2006). Furthermore, in Vegfc-deficient mouse embryos at E9.5, we observed decreased branching of cranial vessels as well a shortening of ISVs (supplementary material Fig. $\mathrm{S} 5 \mathrm{~A}, \mathrm{~B})$, similar to zebrafish embryos injected with Vegfc MO (supplementary material Fig. S5C-F), confirming an important conserved role for vegfc in developmental angiogenesis. However, ISVs appeared normal in vegf $c^{u m 18}$ mutants, suggesting that this truncated form of Vegfc might be sufficient for angiogenesis. To further determine the effect of this mutation on angiogenesis, we assessed ISV formation in vegf $c^{u m 18}$ mutant embryos in which this process was mildly inhibited or enhanced.

Zebrafish embryos mutant for $k d r l^{17}$, a zebrafish vegf receptor 2 ortholog, show variable ISV defects that are enhanced by reducing flt4 (Covassin et al., 2009; Covassin et al., 2006). We generated $\operatorname{Tg}(f l i 1 a: e g f p)^{y 1}$ zebrafish that were heterozygous for $k d r l^{y 17}$ and vegfc $c^{u m} 18$ and assessed ISV length in their progeny. In vegf $c^{u m 18 / u m 18}$ homozygous mutant embryos with wild-type $k d r l$, ISV length was normal (Fig. 2A,B,E). However, we noted a slight and significant decrease in ISV length in $k d r l^{+/ y 17}$; veg $f^{+/ u m 18}$ double-heterozygous embryos that was further enhanced by loss of both copies of wildtype vegfc (Fig. 2E). ISVs were significantly shorter in $k d r^{y 17 / y 17}$ mutant embryos that were wild-type for the veg $f$ locus (Fig. 2C,E), with a progressive decrease in $k d r l^{y 17 / y 17} ;$ vegf $c^{+/ u m 18}$ (Fig. 2E) and $k d r l^{\text {y17/y17 }}$; vegfc $c^{\text {um18/um18 }}$ double-mutant embryos (Fig. 2C-E). We also observed ISVs in vegfc $c^{u m 18}$ embryos lacking the Notch ligand, delta-like 4 (dll4), which causes increased ISV endothelial cell number and branching (Leslie et al., 2007; Siekmann and Lawson, 2007) (Fig. 3A,B,G,H). These defects were similar in vegfc ${ }^{u m 18 /+}$ heterozygous embryos lacking dll4 (Fig. 3C,D), although slightly less severe (Fig. 3G,H). Likewise, vegf $c^{u m 18}$ homozygous mutant embryos displayed milder increases in branching and no increase in cell number following D114 knockdown when compared with wild-type siblings (Fig. 3B,E-H). Taken together, these observations suggest that the vegfc ${ }^{\text {um } 18}$ mutation has a mild effect on ISV formation that is evident on a sensitized genetic background.

\section{Vegfc ${ }^{\text {um18 }}$ is not efficiently secreted}

To investigate the effect of the um 18 truncation on the Vegfc protein, we first determined whether Vegfc ${ }^{\text {um } 18}$ could activate Flt4. We generated conditioned medium from Drosophila S2 cells expressing either Vegfc or Vegfc ${ }^{\text {um18 }}$ fused to the BiP secretion signal to reduce possible effects of the C-terminal truncation on secretion. In cells expressing zebrafish Flt4, both Vegfc- and Vegfc ${ }^{\text {um } 18}$-conditioned media induced receptor tyrosine phosphorylation to similar levels (Fig. 4A). Furthermore, injection of Vegfc- or Vegfc ${ }^{\text {um } 18}$ conditioned medium into the perivitelline space of zebrafish embryos caused similar levels of ectopic subintestinal vessel (SIV) formation, whereas mock conditioned medium did not (Fig. 4B-E).

We next examined the effect of um 18 on Vegfc secretion. Since an appropriate antibody against zebrafish Vegfc was not available, we used the human equivalent of Vegfc ${ }^{\mathrm{um} 18}$ (VEGFC ${ }^{\mathrm{um} 18}$ ). In this case, constructs contained the native VEGFC secretion signal. We detected both unprocessed and processed forms of VEGFC in lysates and medium from transfected cells (Fig. 4F). By contrast, VEGFC $^{\text {um } 18}$ accumulated in cell lysates and was not in the medium at 30 hours post-transfection (Fig. 4F,G), although it was detectable by 72 hours (data not shown). We did not detect changes in protein stability caused by the loss of the C-terminal domain in these cases (supplementary material Fig. S6A,B). Interestingly, the furin-
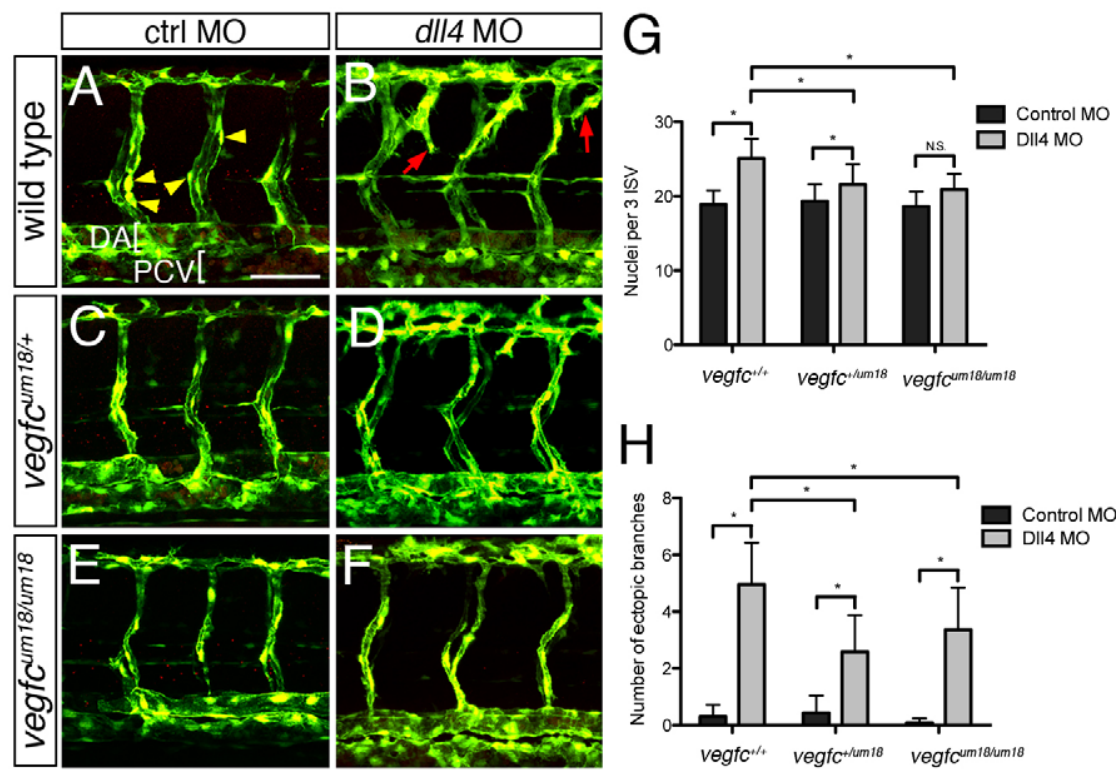

Fig. 3. vegfc ${ }^{u m 18}$ partially rescues loss of $d / 14$.

(A-F) Two-photon micrographs of trunk blood vessels in fixed Tg(fli1a:egfp $)^{y 1}$ zebrafish embryos at $48 \mathrm{hpf}$ immunostained for Fli1 b. Anterior is to the left, dorsal is up. Yellow arrowheads indicate endothelial nuclei. Red arrows indicate ectopic vessel branches. Wild-type $(A, B)$, vegfc $c^{\text {um } 18 /++}(C, D)$ and vegfc um 18/um18 (E,F) embryos were injected with $15 \mathrm{ng}$ control $\mathrm{MO}(\mathrm{A}, \mathrm{C}, \mathrm{E})$ or $15 \mathrm{ng}$ DII4 MO (B,D,F). (G,H) Quantification of $(G)$ nuclei and $(\mathrm{H})$ ectopic branches across three ISVs in MO-injected embryos of the indicated genotype. Values are the average of three experiments. ${ }^{*} P<0.05$; N.S., not significant; error bars indicate s.e.m. DA, dorsal aorta; PCV, posterior cardinal vein. Scale bar: $50 \mu \mathrm{m}$. 
resistant form of Vegfc that is capable of partially rescuing vegf $c^{\text {um } 18}$ lymphatic defects is secreted, despite its inability to be cleaved (supplementary material Fig. S4B,C). To further investigate the effect of the observed secretion defect in vivo, we assessed the ability of xenografted cells expressing zebrafish vegf $c^{\text {um } 18}$ to induce neovascularization in zebrafish embryos. At 2 days post-graft, cells expressing wild-type Vegfc-2Amcherry were highly vascularized whereas those expressing Vegfc ${ }^{\text {um1 } 18}-2$ Amcherry were not (Fig. 4H$\mathrm{J})$. Together, these observations suggest that the um 18 mutation in Vegfc prevents its efficient secretion, but not its ability to activate Flt4.

\section{An endothelial cell-autonomous role for Vegfc during angiogenesis}

$v e g f c$ is expressed in the hypochord, which lies adjacent to the dorsal aorta, as well as in aortic endothelial cells themselves (Covassin et al., 2006). We further find that vegfc is expressed in ISV endothelial cells as they sprout from the aorta (supplementary material Fig. S6C), suggesting that vegfc might play an endothelial cell-autonomous role during angiogenesis. To investigate this possibility, we first determined the effect of Vegfc and Vegfc ${ }^{\text {um18 }}$ on ISVs by overexpressing them in endothelial cells. Transient endothelial expression of either vegfc or $v e g f c^{u m 18}$ in ISVs induced ectopic ISV branching into the horizontal myoseptum, whereas egfp-2Amcherry did not (Fig. 5A-D). Although veg $f c^{u m 18}$ elicited a more variable effect, this difference was not statistically significant
(Fig. 5D). This effect is in contrast to the inability of vegfc $c^{u m 18}$ to rescue lymphatic development (see Fig. 1G,I).

We next determined the endothelial cell-autonomous effect of $v e g f c$ deficiency on ISV growth by performing mosaic analysis. We transplanted cells from donor $\operatorname{Tg}(\text { flila:egfp })^{y l}$ embryos injected with $5 \mathrm{ng}$ Vegfc or control MO, or derived from an incross of vegf $c^{u m} 18$ heterozygous carriers, into $\mathrm{Tg}(k d r l \text { :rasmcherry })^{586}$ host embryos and assessed their positions in trunk blood vessels at $30 \mathrm{hpf}$. Control donor endothelial cells contributed to all trunk blood vessel positions with relatively equal frequency (Fig. 5E,G). By contrast, vegfc morphant cells displayed less frequent contribution to both the distal tip cell position and posterior cardinal vein than control morphant cells (Fig. 5F,G). Although we noted a similar trend in the behavior of vegfc $c^{\text {um } 18}$ mutant cells, the differences were not statistically significant (Fig. $5 \mathrm{H}$ ). Together, these observations demonstrate that vegfc acts in an endothelial cell-autonomous manner to promote ISV growth.

\section{Vegfc is required for persistent forward migration during angiogenesis}

To investigate cellular mechanisms that might be affected by a putative Vegfc/Flt4 autocrine loop, we incorporated these signals into a previously described agent-based computational model (Bentley et al., 2008; Bentley et al., 2009). We then simulated sprouting with Vegfc acting in two possible ways: (1) through a direct effect on Notch signaling and dll4 levels or (2) through a
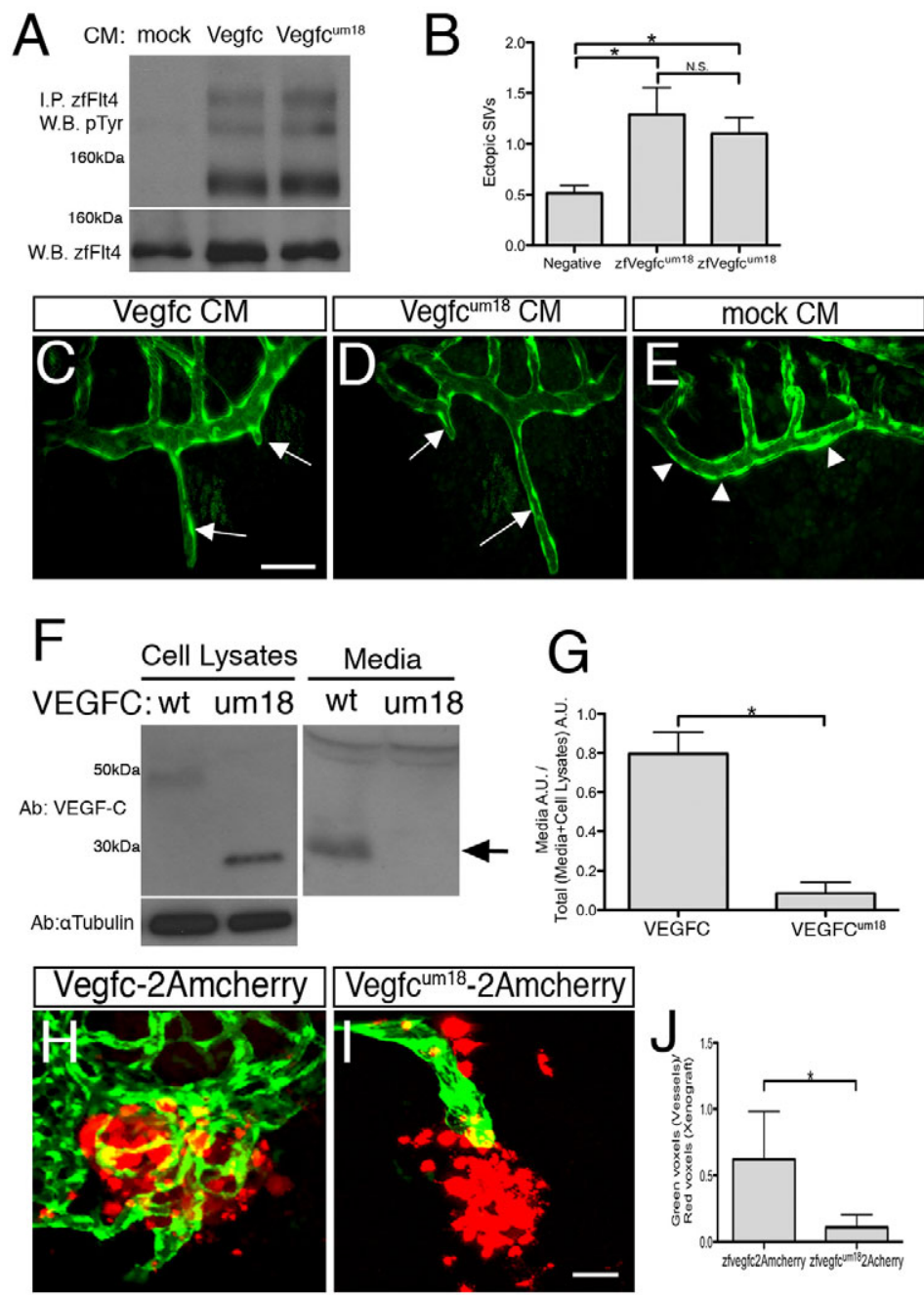
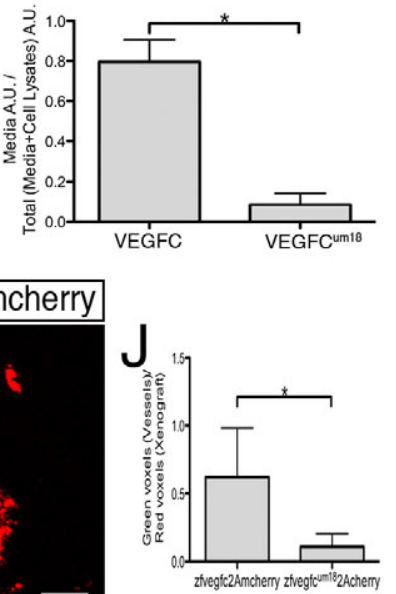

Fig. 4. The um 18 mutation affects Vegfc secretion.

(A) Western blot (W.B.) of Flt4 immunoprecipitates (I.P.) from cells treated with the indicated conditioned medium (CM), probed with antibodies against phosphotyrosine (pTyr) and zebrafish Flt4. (B) Quantification of ectopic subintestinal vessels (SIVs) in embryos injected with the indicated CM. Values are the average of three experiments. (C-E) Confocal micrographs of SIVs (arrowheads) in Tg(fli 1 a:egfp) ${ }^{y 1}$ embryos at $3.5 \mathrm{dpf}$ following perivitelline injections with (C) Vegfc $C M$, (D) Vegfc ${ }^{\text {um } 18} \mathrm{CM}$ or (E) mock CM. Arrows indicate ectopic SIVs. (F) Western blots probed with the indicated antibodies. Cell lysates and media from $\mathrm{NIH} 3 \mathrm{~T} 3$ cells transiently expressing human VEGFC or VEGFCum18. Arrow indicates processed secreted form of wild-type VEGFC.

(G) Quantitation of western blots in F. Values are the average of three experiments. A.U., arbitrary units. $(\mathbf{H}, \mathbf{I})$. Confocal image of xenografts in 4.5-dpf Tg(fli la:egfp) ${ }^{y 1}$ embryos expressing $(\mathrm{H})$

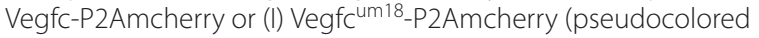
red). (J) Quantification of vascular density in xenografts from three experiments. $(\mathrm{C}-\mathrm{E}, \mathrm{H}, \mathrm{I})$ Anterior is to the left, dorsal is up. ${ }^{*} P<0.05$; N.S., not significant; error bars indicate s.e.m. Scale bars: $50 \mu \mathrm{m}$ in $\mathrm{C}-\mathrm{E} ; 10 \mu \mathrm{m}$ in $\mathrm{H}, \mathrm{I}$. 

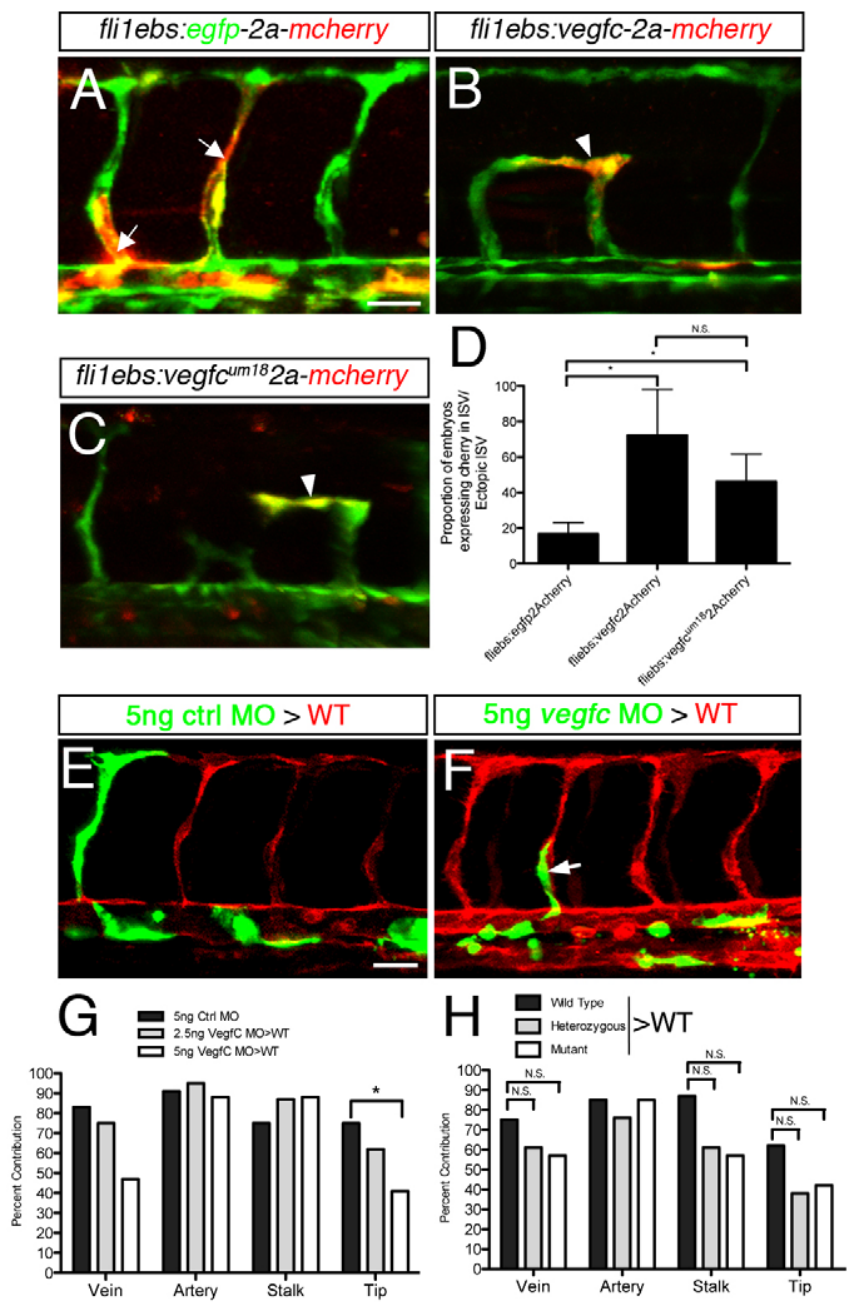

Fig. 5. Endothelial cell-autonomous effects of Vegfc on angiogenesis. (A-C) Confocal micrographs of trunk blood vessels in $\mathrm{Tg}$ (fli1a:egfp) $)^{y 1}$ zebrafish embryos at 30 hpf. Anterior to the left, dorsal is up. Transgene expression (red fluorescence) is indicated in normal (arrow) or ectopically branched (arrowheads) ISVs. Embryos were co-injected with tol2 transposase mRNA and (A) pTol2-fliebs:egfp-2Acherry, (B) pTol2fliebs:vegfc-2Acherry or (C) pTol2fliebs:vegfc um 18-2Acherry. (D) Quantification of ectopic ISV branching in cells expressing the fliebs:egfp-2Acherry $(n=12$ embryos) or fliebs:vegfc-2Acherry ( $n=18$ embryos) transgene. Values indicate the proportion of embryos with Cherry-positive ISVs displaying ectopic branching. (E,F) Confocal micrographs of trunk blood vessels at 30 hpf of $T g$ (kdrl:rasmcherry) ${ }^{5896}$ host embryos transplanted with

$\mathrm{Tg}$ (fli1a:egfp) ${ }^{y 1}$ cells (green) from embryos injected with $5 \mathrm{ng}(\mathrm{E})$ control $\mathrm{MO}$ or (F) Vegfc MO. Arrow indicates a stalk cell. $(\mathbf{G}, \mathbf{H})$ The proportion of embryos displaying donor cells in the indicated trunk vessel position. $(\mathrm{G})$ Donor cells from embryos injected with the indicated $\mathrm{MO}$. $(\mathrm{H})$ Donor cells were from embryos derived from an incross of vegfc ${ }^{\text {um } 18}$ carriers. ${ }^{*} P<0.05$; N.S., not significant; error bars indicate s.e.m. Scale bars: $50 \mu \mathrm{m}$.

direct effect on the migratory apparatus, with indirect effects on Notch. Only the latter mechanism yielded an output similar to both the experimentally derived transplantation and ISV length data. Simulating a direct effect on Notch failed to recapitulate ISV growth defects, although it could mimic the transplantation data (Fig. 6A; supplementary material Table S2 and Fig. S7A). In particular, the experimental data could be matched by specifically destabilizing filopodia in vegfc-deficient cells. Accordingly, a higher chance of random filopodia retraction led to a migratory defect in which $v e g f c$ deficient cells lagged behind control cells in simulations of growing ISVs (Fig. 6A; supplementary material Movies 1,2). Cells lacking $v e g f c$ also showed a moderately reduced ability to contribute to the tip cell position in competition simulations, consistent with mosaic analyses (supplementary material Table S2 and Movies 3, 4). These results suggest that a decrease in filopodia persistence might cause slower ISV growth rates in Vegfc-deficient embryos.

To test the simulation predictions, we performed two-photon time-lapse analysis of ISVs at 5-minute intervals in $T g(f l i l a: e g f p)^{y 1}$ embryos injected with $5 \mathrm{ng}$ control or Vegfc MO. We focused on the first few hours of ISV growth to eliminate secondary effects resulting from ISVs being at different positions relative to surrounding pro-angiogenic growth factors (e.g. Vegfa) in control and Vegfc-deficient embryos at later time points. This analysis revealed that ISVs grew more slowly in embryos injected with Vegfc MO, which is qualitatively consistent with growth curves generated by the simulations (Fig. 6A,B). We also noted a slight reduction in the average time of filopodia persistence in vegfcdeficient embryos (13.27 minutes in control MO-injected embryos versus 10.14 minutes in vegfc-deficient embryos; Fig. $6 \mathrm{C}$ ) as predicted by the simulations, although this fell just under the cut-off for statistical significance $(P=0.0501)$. We observed that early ISV growth was characterized by both forward migration and retraction back towards the dorsal aorta in control MO- and Vegfc MOinjected embryos (supplementary material Movies 5, 6). Whereas the frequency of forward migration or retraction was unchanged (data not shown), the average magnitude of retraction, but not forward movement, was significantly greater in ISVs lacking vegfc compared with the control (Fig. 6D,E). Interestingly, we noted similar behaviors in simulations of growing tip cells during competition (compare supplementary material Movie 3 and Movie 4). Thus, vegfc appears to contribute to the persistent forward migration of ISVs and may also affect filopodial stability during angiogenesis.

\section{DISCUSSION}

Vegfc is an essential regulator of lymphatic vessel development (Hogan et al., 2009; Karkkainen et al., 2004; Tammela et al., 2008). However, less is known about its role in developmental angiogenesis or about the relative importance of distinct Vegfc domains for its function. Here, we find that vegfc is important for endothelial tip cell migration during angiogenesis, consistent with the proposed role of its receptor flt4 (Siekmann et al., 2008). Our observations further suggest that vegfc functions in an endothelial cell-autonomous manner in this regard. Indeed, veg $f c$ is expressed in ISVs as they sprout from the dorsal aorta and mosaic analysis demonstrates that vegfc-deficient endothelial cells are less likely to contribute to the tip cell position in ISVs. These results are consistent with the ISV growth defects observed in rspol mutant zebrafish, which lack endothelial vegfc expression, and can be rescued with an endothelial cell-specific vegfc transgene (Gore et al., 2011). Interestingly, ISV tip cells in rspol mutant embryos displayed significantly fewer filopodia, consistent with our observation that Vegfc contributes to filopodia stability.

Co-expression of $f l t 4$ and $v e g f c$ in ISVs and the cell-autonomous requirement for vegfc suggests that a Vegfc/Flt4 autocrine loop is important to promote tip cell migration and angiogenesis (supplementary material Fig. S7B). Vascular endothelial growth factor A (Vegfa) can also act in an autocrine loop to promote endothelial cell survival (Lee et al., 2007). However, we did not observe overt indications of apoptosis in vegfc-deficient embryos 

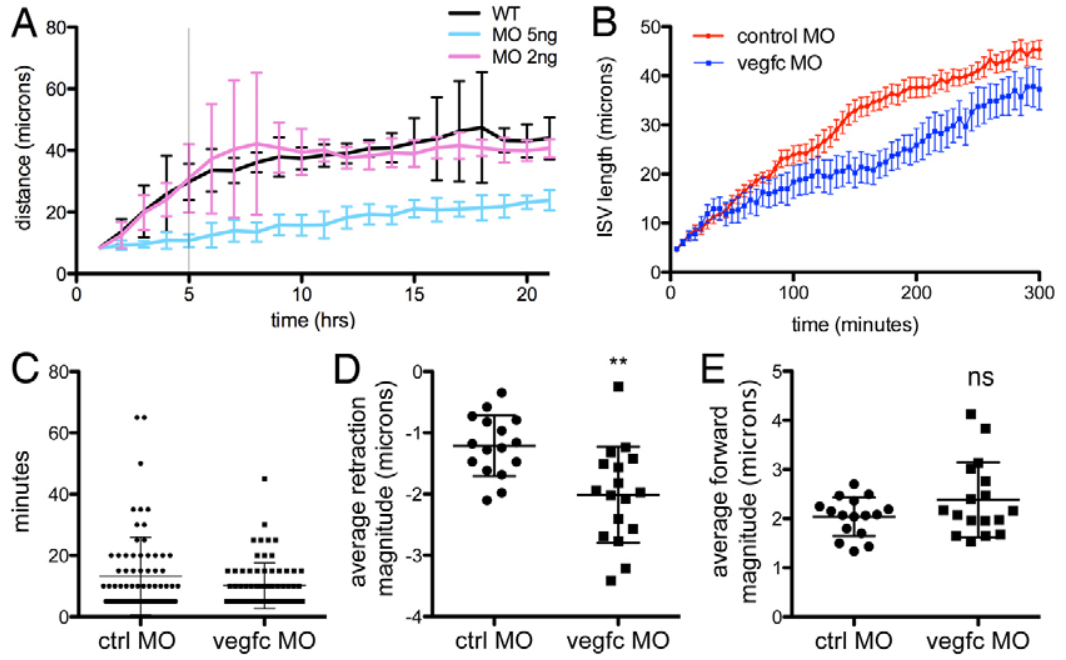

Fig. 6. Vegfc is required for directional persistence.

(A) ISV growth in computational simulations of wild-type or Vegfc MO-injected zebrafish embryos. (B) Difference in ISV growth rate from two-photon time-lapse analysis of $\mathrm{Tg}$ (fli la:egfp) ${ }^{y 1}$ embryos injected with 5 ng control or Vegfc MO. Each time point is an average of 12 (control MO) or 13 (Vegfc MO) ISVs measured at 5-minute intervals from at least four embryos. The difference in the means across all intervals was significant by a paired $t$ test $(P<0.0001)$. (C) Filopodia persistence (measured in minutes). Measurements were derived from 75 filopodia counted across five control or Vegfc MO-injected embryos in parallel 50-minute intervals of two-photon time-lapse movies. (D) Average magnitude of ISV retraction. Data were obtained from at least 16 separate ISVs observed in two-photon time-lapse movies of at least six embryos. ${ }^{*} P=0.015$. (E) Average magnitude of ISV forward migration. Data were obtained from the same ISVs as in D. ns, not significant. Error bars indicate s.e.m. (data not shown). Rather, our data suggest that autocrine Vegfc/Flt4 signaling is required for endothelial migration and directional persistence. Epidermal growth factor (Egf) functions in a similar manner during epithelial cell migration (Maheshwari et al., 2001), in which autocrine signaling maintains directional persistence. Moreover, a truncated form of Egf that is retained within the cell can promote migration in an intracrine manner. This observation suggests that the lack of overt angiogenesis defects in vegfc $c^{\text {um } 18}$ mutant embryos might be due to compensatory intracrine signaling as the truncated protein would be capable of activating co-expressed Flt4 within the cell. Indeed, endothelial-expressed vegfc $c^{\text {um } 18}$ can induce ectopic ISV branching despite its failure to rescue lymphatic development, where it acts as a paracrine factor. However, ISV formation is affected in vegfc ${ }^{u m 18}$ embryos lacking either $k d r l$ or dll4, suggesting that intracrine signaling might not be optimal. An intracrine mechanism might also explain differences in ISV defects between $v e g f c^{u m 18}$ mutants and other examples of vegfc deficiency in zebrafish, which can more severely affect ISV sprouting (e.g. vegfc morphants or rspol mutant embryos). Although our results suggest that a Vegfc/Flt4 autocrine loop promotes angiogenesis, we cannot rule out possible paracrine roles. Indeed, macrophageexpressed $V e g f c$ is thought to play a central role in postnatal mouse retinal angiogenesis (Tammela et al., 2011). Thus, further work using cell type-specific ablation of vegfc will be essential to better define the signaling modalities in each of these contexts.

The memAgent-Spring computational model has previously proved insightful for revealing the importance of a Vegf-Notch feedback loop in modulating endothelial tip and stalk cell behaviors (Bentley et al., 2008; Bentley et al., 2009). Simulations using this model correctly predicted that cells within a growing sprout would continuously compete for the tip cell position and that the 'winner' is determined by relative levels of Vegf and Notch (Jakobsson et al., 2010). However, these simulations had not previously incorporated Vegfc or its receptor. Our integration of a Vegfc/Flt4 autocrine loop into this model suggested an important role for this pathway in ISV growth, filopodia stability and directional persistence. Accordingly, we observed in vivo evidence for the model-predicted effects on ISV growth by time-lapse analyses. Although ISV growth defects following Vegfc knockdown were somewhat milder in vivo than those predicted by the model, these quantitative discrepancies are expected because the output from the model simulations is necessarily qualitative; as in most computational models, the input parameters are clearly limited to the known factors under investigation in this study. Furthermore, detailed quantitative values, such as the number of receptor molecules per cell or receptor/ligand binding affinities, are currently lacking and so could not be included in the model. Nevertheless, both the model and in vivo observations point toward an important role for Vegfc in maintaining filopodia stability and directional persistence during angiogenesis. Importantly, this mechanism might not otherwise have been identified without the use of the memAgent-Spring model and resulting predictions gained from its application.

Whereas angiogenesis is mildly affected by the $u m 18$ mutation, lymphatic development is severely blocked, with defects even in heterozygous embryos. These phenotypes are consistent with the inability of the mutant protein to be efficiently secreted, underscoring the function of Vegfc as a paracrine factor that directs lymphatic development. In mouse embryos, Vegfc from mesenchymal cells adjacent to the cardinal vein promotes the sprouting of lymphangioblasts (Karkkainen et al., 2004). In zebrafish, venous sprouts that will give rise to the lymphatic system likewise grow towards Vegfc, which is expressed in the endothelial cells of the dorsal aorta. Interestingly, the arterial vasculature in the zebrafish guides patterning of the developing lymphatic system (Bussmann et al., 2010). Given that vegfc is specifically expressed in arterial endothelial cells and that its secretion is essential for lymphatic development, our results support a model in which arteries guide the development of nascent lymph vessels, in part, through the paracrine action of Vegfc (supplementary material Fig. S7B).

The um 18 mutation eliminates the Vegfc C-terminal domain, which contains tandemly repeated cysteine-rich motifs resembling those of the silk protein BR3P (Kukk et al., 1996). Domain-swap studies have demonstrated that the C-terminal domain could potentiate angiogenic responses when fused to Vegfa (Keskitalo et al., 2007). Conversely, fusion of the Vegfc VEGF homology domain (VHD) to the Vegfa C-terminus limited its ability to induce lymphatic vessel growth when compared with wild-type Vegfc (Tammela et al., 2007). Whereas these studies suggested a possible structural role for the Vegfc C-terminal domain within the extracellular matrix, our work implicates this domain in secretion. Previous studies reported that the Vegfc VHD alone can be secreted from cells. Indeed, we observe that Vegfc ${ }^{\text {um } 18}$ can eventually be 
found in the medium from transfected cells. However, there is a clear defect in secretion and a vegf $c^{\text {um } 18}$ transgene is not capable of rescuing lymphatic development in zebrafish embryos. It is likely that eventual secretion in cell culture experiments is due to high levels of transgene expression driven by constitutively highly active promoters. Furthermore, many constructs from previous studies contain heterologous secretion signals that might mask the importance of the C-terminus. Thus, we believe that our results support an important role for the Vegfc C-terminal domain in secretion, although the intervening amino acid sequences between the um 18 truncation and the furin cleavage site might also be important. How these domains act to promote efficient transport through the secretory apparatus is currently unclear. Given the ease with which transient rescue experiments can be performed in the vegf $c^{\text {um } 18}$ mutants, it will be possible to further dissect the importance of this and other domains within Vegfe for its function during lymphatic development.

\section{Acknowledgements}

We thank John Polli and Ed Jaskolski for excellent fish care; Julie Zhu for advice concerning statistical analyses; and Tom Smith and George Kourkoulis for technical assistance.

\section{Funding}

This work was funded by National Institutes of Health grants [R01HL101374 and R01HL093467] from the National Heart, Lung, and Blood Institute to N.D.L. J.A.V. was supported by a Ruth L. Kirschstein pre-doctoral National Research Service Award to promote diversity in health-related research [5F31HL081927]. H.G. and K.B. are funded by Cancer Research UK, the Leducq Transatlantic Network ARTEMIS, and the Lister Institute of Preventive Medicine. Deposited in PMC for release after 12 months.

\section{Competing interests statement}

The authors declare no competing financial interests.

\section{Supplementary material}

Supplementary material available online at

http://dev.biologists.org/lookup/suppl/doi:10.1242/dev.084152/-/DC1

\section{References}

Benedito, R., Rocha, S. F., Woeste, M., Zamykal, M., Radtke, F., Casanovas, O., Duarte, A., Pytowski, B. and Adams, R. H. (2012). Notch-dependent VEGFR3 upregulation allows angiogenesis without VEGF-VEGFR2 signalling. Nature 484, 110-114

Bentley, K., Gerhardt, H. and Bates, P. A. (2008). Agent-based simulation of notch-mediated tip cell selection in angiogenic sprout initialisation. J. Theor. Biol. 250, 25-36.

Bentley, K., Mariggi, G., Gerhardt, H. and Bates, P. A. (2009). Tipping the balance: robustness of tip cell selection, migration and fusion in angiogenesis. PLoS Comput. Biol. 5, e1000549.

Bussmann, J., Bos, F. L., Urasaki, A., Kawakami, K., Duckers, H. J. and Schulte-Merker, S. (2010). Arteries provide essential guidance cues for lymphatic endothelial cells in the zebrafish trunk. Development 137, 26532657.

Cleaver, O. and Krieg, P. A. (2010). Vascular development. In Heart Development and Regeneration (ed. N. Rosenthal and R. P. Harvey), pp. 487-528. London: Elsevier.

Covassin, L. D., Villefranc, J. A., Kacergis, M. C., Weinstein, B. M. and Lawson, N. D. (2006). Distinct genetic interactions between multiple Vegf receptors are required for development of different blood vessel types in zebrafish. Proc. Natl. Acad. Sci. USA 103, 6554-6559.

Covassin, L. D., Siekmann, A. F., Kacergis, M. C., Laver, E., Moore, J. C., Villefranc, J. A., Weinstein, B. M. and Lawson, N. D. (2009). A genetic screen for vascular mutants in zebrafish reveals dynamic roles for Vegf/PIcg1 signaling during artery development. Dev. Biol. 329, 212-226.

Dumont, D. J., Jussila, L., Taipale, J., Lymboussaki, A., Mustonen, T., Pajusola, K., Breitman, M. and Alitalo, K. (1998). Cardiovascular failure in mouse embryos deficient in VEGF receptor-3. Science 282, 946-949.

Gore, A. V., Swift, M. R., Cha, Y. R., Lo, B., McKinney, M. C., Li, W., Castranova, D., Davis, A., Mukouyama, Y. S. and Weinstein, B. M. (2011). Rspo1/Wnt signaling promotes angiogenesis via Vegfc/Negfr3. Development 138, 48754886.

Herbert, S. P., Huisken, J., Kim, T. N., Feldman, M. E., Houseman, B. T., Wang, R. A., Shokat, K. M. and Stainier, D. Y. (2009). Arterial-venous segregation by selective cell sprouting: an alternative mode of blood vessel formation. Science 326, 294-298.

Hogan, B. M., Herpers, R., Witte, M., Heloterä, H., Alitalo, K., Duckers, H. J. and Schulte-Merker, S. (2009). Vegfc/Flt4 signalling is suppressed by Dll4 in developing zebrafish intersegmental arteries. Development 136, 4001-4009.

Isogai, S., Horiguchi, M. and Weinstein, B. M. (2001). The vascular anatomy of the developing zebrafish: an atlas of embryonic and early larval development. Dev. Biol. 230, 278-301.

Jakobsson, L., Franco, C. A., Bentley, K., Collins, R. T., Ponsioen, B., Aspalter, I. M., Rosewell, I., Busse, M., Thurston, G., Medvinsky, A. et al. (2010) Endothelial cells dynamically compete for the tip cell position during angiogenic sprouting. Nat. Cell Biol. 12, 943-953.

Jeltsch, M., Kaipainen, A., Joukov, V., Meng, X., Lakso, M., Rauvala, H., Swartz, M., Fukumura, D., Jain, R. K. and Alitalo, K. (1997). Hyperplasia of lymphatic vessels in VEGF-C transgenic mice. Science 276, 1423-1425.

Joukov, V., Pajusola, K., Kaipainen, A., Chilov, D., Lahtinen, I., Kukk, E., Saksela, O., Kalkkinen, N. and Alitalo, K. (1996). A novel vascular endothelial growth factor, VEGF-C, is a ligand for the Flt4 (VEGFR-3) and KDR (VEGFR-2) receptor tyrosine kinases. EMBO J. 15, 290-298.

Joukov, V., Sorsa, T., Kumar, V., Jeltsch, M., Claesson-Welsh, L., Cao, Y., Saksela, O., Kalkkinen, N. and Alitalo, K. (1997). Proteolytic processing regulates receptor specificity and activity of VEGF-C. EMBO J. 16, 3898-3911.

Kaipainen, A., Korhonen, J., Mustonen, T., van Hinsbergh, V. W., Fang, G. H., Dumont, D., Breitman, M. and Alitalo, K. (1995). Expression of the fms-like tyrosine kinase 4 gene becomes restricted to lymphatic endothelium during development. Proc. Natl. Acad. Sci. USA 92, 3566-3570.

Karkkainen, M. J., Ferrell, R. E., Lawrence, E. C., Kimak, M. A., Levinson, K. L., McTigue, M. A., Alitalo, K. and Finegold, D. N. (2000). Missense mutations interfere with VEGFR-3 signalling in primary lymphoedema. Nat. Genet. 25, 153-159.

Karkkainen, M. J., Haiko, P., Sainio, K., Partanen, J., Taipale, J., Petrova, T. V., Jeltsch, M., Jackson, D. G., Talikka, M., Rauvala, H. et al. (2004). Vascular endothelial growth factor $C$ is required for sprouting of the first lymphatic vessels from embryonic veins. Nat. Immunol. 5, 74-80.

Keskitalo, S., Tammela, T., Lyytikka, J., Karpanen, T., Jeltsch, M., Markkanen, J., Yla-Herttuala, S. and Alitalo, K. (2007). Enhanced capillary formation stimulated by a chimeric vascular endothelial growth factor/vascular endothelial growth factor-C silk domain fusion protein. Circ. Res. 100, 14601467

Küchler, A. M., Gjini, E., Peterson-Maduro, J., Cancilla, B., Wolburg, H. and Schulte-Merker, S. (2006). Development of the zebrafish lymphatic system requires VEGFC signaling. Curr. Biol. 16, 1244-1248.

Kukk, E., Lymboussaki, A., Taira, S., Kaipainen, A., Jeltsch, M., Joukov, V. and Alitalo, K. (1996). VEGF-C receptor binding and pattern of expression with VEGFR-3 suggests a role in lymphatic vascular development. Development 122, 3829-3837.

Lawson, N. D. and Weinstein, B. M. (2002). In vivo imaging of embryonic vascular development using transgenic zebrafish. Dev. Biol. 248, 307-318.

Lawson, N. D., Scheer, N., Pham, V. N., Kim, C. H., Chitnis, A. B., CamposOrtega, J. A. and Weinstein, B. M. (2001). Notch signaling is required for arterial-venous differentiation during embryonic vascular development. Development 128, 3675-3683.

Lawson, N. D., Mugford, J. W., Diamond, B. A. and Weinstein, B. M. (2003) phospholipase $\mathrm{C}$ gamma-1 is required downstream of vascular endothelial growth factor during arterial development. Genes Dev. 17, 1346-1351.

Lee, S., Chen, T. T., Barber, C. L., Jordan, M. C., Murdock, J., Desai, S., Ferrara, N., Nagy, A., Roos, K. P. and Iruela-Arispe, M. L. (2007). Autocrine VEGF signaling is required for vascular homeostasis. Cell 130, 691-703.

Leslie, J. D., Ariza-McNaughton, L., Bermange, A. L., McAdow, R., Johnson, S. L. and Lewis, J. (2007). Endothelial signalling by the Notch ligand Delta-like 4 restricts angiogenesis. Development 134, 839-844.

Lohela, M., Heloterä, H., Haiko, P., Dumont, D. J. and Alitalo, K. (2008). Transgenic induction of vascular endothelial growth factor-C is strongly angiogenic in mouse embryos but leads to persistent lymphatic hyperplasia in adult tissues. Am. J. Pathol. 173, 1891-1901.

Maheshwari, G., Wiley, H. S. and Lauffenburger, D. A. (2001). Autocrine epidermal growth factor signaling stimulates directionally persistent mammary epithelial cell migration. J. Cell Biol. 155, 1123-1128.

Nicoli, S., De Sena, G. and Presta, M. (2009). Fibroblast growth factor 2induced angiogenesis in zebrafish: the zebrafish yolk membrane (ZFYM) angiogenesis assay. J. Cell. Mol. Med. 13, 2061-2068.

Nicoli, S., Standley, C., Walker, P., Hurlstone, A., Fogarty, K. E. and Lawson, N. D. (2010). MicroRNA-mediated integration of haemodynamics and Vegf signalling during angiogenesis. Nature 464, 1196-1200.

Nicoli, S., Knyphausen, C. P., Zhu, L. J., Lakshmanan, A. and Lawson, N. D. (2012). miR-221 is required for endothelial tip cell behaviors during vascular development. Dev. Cell 22, 418-429.

Poole, T. J. and Coffin, J. D. (1989). Vasculogenesis and angiogenesis: two distinct morphogenetic mechanisms establish embryonic vascular pattern. J. Exp. Zool. 251, 224-231. 
Provost, E., Rhee, J. and Leach, S. D. (2007). Viral 2A peptides allow expression of multiple proteins from a single ORF in transgenic zebrafish embryos. Genesis 45, 625-629.

Siegfried, G., Basak, A., Cromlish, J. A., Benjannet, S., Marcinkiewicz, J. Chrétien, M., Seidah, N. G. and Khatib, A. M. (2003). The secretory proprotein convertases furin, PC5, and PC7 activate VEGF-C to induce tumorigenesis. J. Clin. Invest. 111, 1723-1732.

Siekmann, A. F. and Lawson, N. D. (2007). Notch signalling limits angiogenic cell behaviour in developing zebrafish arteries. Nature 445, 781-784

Siekmann, A. F., Covassin, L. and Lawson, N. D. (2008). Modulation of VEGF signalling output by the Notch pathway. BioEssays 30, 303-313.

Srinivasan, R. S., Dillard, M. E., Lagutin, O. V., Lin, F. J., Tsai, S., Tsai, M. J., Samokhvalov, I. M. and Oliver, G. (2007). Lineage tracing demonstrates the venous origin of the mammalian lymphatic vasculature. Genes Dev. 21, 24222432.

Tammela, T. and Alitalo, K. (2010). Lymphangiogenesis: molecular mechanisms and future promise. Cell 140, 460-476.

Tammela, T., He, Y., Lyytikkä, J., Jeltsch, M., Markkanen, J., Pajusola, K., YläHerttuala, S. and Alitalo, K. (2007). Distinct architecture of lymphatic vessels induced by chimeric vascular endothelial growth factor-C/vascular endothelial growth factor heparin-binding domain fusion proteins. Circ. Res. 100, 1468-1475.

Tammela, T., Zarkada, G., Wallgard, E., Murtomäki, A., Suchting, S., Wirzenius, M., Waltari, M., Hellström, M., Schomber, T., Peltonen, R. et al. (2008). Blocking VEGFR-3 suppresses angiogenic sprouting and vascular network formation. Nature 454, 656-660

Tammela, T., Zarkada, G., Nurmi, H., Jakobsson, L., Heinolainen, K. Tvorogov, D., Zheng, W., Franco, C. A., Murtomäki, A., Aranda, E. et al. (2011). VEGFR-3 controls tip to stalk conversion at vessel fusion sites by reinforcing Notch signalling. Nat. Cell Biol. 13, 1202-1213.

Veikkola, T., Jussila, L., Makinen, T., Karpanen, T., Jeltsch, M., Petrova, T. V., Kubo, H., Thurston, G., McDonald, D. M., Achen, M. G. et al. (2001). Signalling via vascular endothelial growth factor receptor-3 is sufficient for lymphangiogenesis in transgenic mice. EMBO J. 20, 1223-1231.

Villefranc, J. A., Amigo, J. and Lawson, N. D. (2007). Gateway compatible vectors for analysis of gene function in the zebrafish. Dev. Dyn. 236, 3077-3087.

Yaniv, K., Isogai, S., Castranova, D., Dye, L., Hitomi, J. and Weinstein, B. M. (2006). Live imaging of lymphatic development in the zebrafish. Nat. Med. 12 711-716. 